\title{
ON THE WELL-POSEDNESS OF THE LINEAR PERIDYNAMIC MODEL AND ITS CONVERGENCE TOWARDS THE NAVIER EQUATION OF LINEAR ELASTICITY*
}

\author{
ETIENNE EMMRICH ${ }^{\dagger}$ AND OLAF WECKNER ${ }^{\ddagger}$
}

\begin{abstract}
The non-local peridynamic theory describes the displacement field of a continuous body by the initial-value problem for an integro-differential equation that does not include any spatial derivative. The non-locality is determined by the so-called peridynamic horizon $\delta$ which is the radius of interaction between material points taken into account.

Well-posedness and structural properties of the peridynamic equation of motion are established for the linear case corresponding to small relative displacements. Moreover the limit behavior as $\delta \rightarrow 0$ is studied.
\end{abstract}

Key words. linear elasticity, non-local theory, peridynamic equation, Navier equation

AMS subject classifications. 35Q72, 74B05, 74B99, 74H10, 74H20, 74H25

\section{Introduction}

The peridynamic model has been introduced in Silling [33] as a non-local elasticity theory in integral form that avoids spatial derivatives. It essentially relies upon differences of the displacement of material points interacting within a prescribed horizon. Here also lies the possible advantage as the evolution of discontinuities in the displacement or the gradient of the displacement might be inherently described within the peridynamic model. Typical applications are the autonomous propagation of cracks of phase boundaries.

Non-local theories taking into account effects of long-range interactions in elastic materials, and their application to problems of solid and fracture mechanics have been studied for a long time, cf. the pioneering work by Kröner [24], Eringen [20] and the references cited therein, the monographs by Kunin [25] and Rogula [32], and more recently (without being exhaustive) Altan [1, 2], Bažant \& Jirásek [5], Chen et al. [13, 12], Lei et al. [27], Pisano \& Fuschi [29], Polizzotto [30, 31], Wang \& Dhaliwal [40, 41].

Different aspects of the peridynamic model such as its theoretical substantiation, its numerical approximation, and its application have been studied in Bobaru et al. [9, 10], Dayal \& Bhattacharya [14], Emmrich \& Weckner [16, 17, 18], Gerstle et al. [22, 23], Silling [34], Silling \& Askari [36, 35], Silling \& Bobaru [37], Silling et al. [38], Weckner \& Abeyaratne [42], Weckner \& Emmrich [43].

Here, we establish new results on well-posedness and dissipativity for the linear peridynamic model. This might be seen as a first step towards the analysis of the nonlinear model that allows, in contrast to the linear case, also large displacements as they occur, e.g. for cracks. Existence and uniqueness follow from interpreting the equation of motion as an abstract ordinary differential equation with a bounded operator in a Lebesgue space. The exact solution can be represented by Duhamel's principle from which then a priori estimates and stability follow. For deriving a

* Received: May 15, 2007; accepted (in revised version): August 29, 2007. Communicated by Claude Le Bris.

${ }^{\dagger}$ Technische Universität Berlin, Institut für Mathematik, Straße des 17. Juni 136, 10623 Berlin, Germany (emmrich@math.tu-berlin.de).

$\ddagger$ The Boeing Company, Mathematics \& Engineering Analysis, P.O. Box 3707, MC 7L-21, Seattle, WA 98124-2207, United States (olaf.weckner@boeing.com). 
priori estimates uniform in the parameter $\delta$ describing the non-locality, an inequality established in Bourgain et al. [11] relating the $H^{1}$-norm of a function to a double integral over divided differences of the function is employed. First results on wellposedness can be found in Emmrich \& Weckner [16, 18].

Moreover, this paper is a first attempt to study the relation between the general linear peridynamic model and the classical Navier equation of linear elasticity. It turns out that the integral operator in the peridynamic equation of motion, which describes the spatial interaction, applied on a smooth function becomes in the limit of vanishing non-locality just the differential operator of the Navier equation. This result can be proven for a large class of linear material ansatzes. However, the convergence only takes place in an interior subdomain of the domain under consideration. This also reflects the fact that there is no place for posing boundary conditions within the peridynamic model as there are no spatial derivatives.

Besides, we prove an expansion of the peridynamic integral operator in terms of higher order spatial derivatives in an interior domain. The proof mainly relies upon a Taylor expansion of the displacement field. From studying particular nonlinear dispersion relations for the one-dimensional infinite peridynamic bar, it is already known that the peridynamic integral operator equals for smooth functions a differential operator of infinite order that is a series of derivatives of even order (cf. Weckner \& Abeyaratne [42]). Again for the infinite peridynamic bar but relying on another material model, it has been shown in Emmrich \& Weckner [16] that the peridynamic integral operator becomes for sufficiently smooth functions the differential operator of the wave equation augmented by a mixed derivative of fourth order (second order in time and space) that describes lateral contraction.

It is interesting to see that Taylor expansions similar to those arising here have also been observed in Arndt \& Griebel [4] in connection with higher-order gradient theories for crystalline solids in the one-dimensional case. The relation between atomistic and continuum models for crystalline solids and corresponding expansions have also been studied in E \& Ming [15]. We also refer to Blanc et al. $[6,7,8]$ and Le Bris \& Lions [26] for a discussion of the relation between molecular models and models on a continuum level.

Throughout this paper, we rely upon the usual notation for Lebesgue- and Bochner-Lebesgue-spaces as well as spaces of continuously differentiable functions. By $C$, we denote a generic positive constant that is independent of $\delta$.

\section{The peridynamic equation of motion}

The governing equation in the peridynamic theory is the second-order in time partial integro-differential equation

$$
\rho(\boldsymbol{x}) \partial_{t}^{2} \boldsymbol{u}(\boldsymbol{x}, t)=\int_{\mathcal{V}} \boldsymbol{f}(\boldsymbol{x}, \hat{\boldsymbol{x}}, \boldsymbol{u}(\boldsymbol{x}, t), \boldsymbol{u}(\hat{\boldsymbol{x}}, t), t) \mathrm{d} \hat{\boldsymbol{x}}+\boldsymbol{b}(\boldsymbol{x}, t), \quad(\boldsymbol{x}, t) \in \mathcal{V} \times(0, T),
$$

in Lagrangian coordinates $\boldsymbol{x}$, where $\rho$ denotes the mass density that shall be bounded away from zero with $1 / \rho \in L^{\infty}(\mathcal{V}), \boldsymbol{u}$ the displacement field of the body that occupies the volume $\mathcal{V} \subseteq \mathbb{R}^{d}(d \in\{1,2,3\}), \boldsymbol{f}$ the pairwise force function that describes the internal forces, and $\boldsymbol{b}$ an inhomogeneity that collects all external forces per unit volume. By $T>0$, the time under consideration is denoted. The volume $\mathcal{V}$ is supposed to be a bounded domain of class $\mathcal{C}^{0,1}$. Equ. (2.1) is supplemented by the initial conditions

$$
\boldsymbol{u}(\cdot, 0)=\boldsymbol{u}_{0}, \quad \partial_{t} \boldsymbol{u}(\cdot, 0)=\dot{\boldsymbol{u}}_{0} .
$$


Note that no boundary conditions appear as there are no spatial derivatives.

In view of the balance of the angular momentum of the mass-free bond between $\boldsymbol{x}$ and $\hat{\boldsymbol{x}}$, the pairwise force function $\boldsymbol{f}$ always points from the current position $\boldsymbol{x}+\boldsymbol{u}(\boldsymbol{x}, t)$ of the point $\boldsymbol{x}$ to the current position $\hat{\boldsymbol{x}}+\boldsymbol{u}(\hat{\boldsymbol{x}}, t)$ of the point $\hat{\boldsymbol{x}}$. Moreover, the invariance against a rigid body motion, the assumption of no explicit time dependence, and Newton's third law lead to $\boldsymbol{f}(\boldsymbol{x}, \hat{\boldsymbol{x}}, \boldsymbol{u}, \hat{\boldsymbol{u}}, t)=\boldsymbol{f}(\boldsymbol{x}, \hat{\boldsymbol{x}}, \hat{\boldsymbol{u}}-\boldsymbol{u})$ with $\boldsymbol{f}(\hat{\boldsymbol{x}}, \boldsymbol{x},-\boldsymbol{\eta})=-\boldsymbol{f}(\boldsymbol{x}, \hat{\boldsymbol{x}}, \boldsymbol{\eta})$ for all $\boldsymbol{x}, \hat{\boldsymbol{x}}, \boldsymbol{\eta}:=\hat{\boldsymbol{u}}-\boldsymbol{u}$. It is typical for the peridynamic model to require

$$
\boldsymbol{f}(\boldsymbol{x}, \hat{\boldsymbol{x}}, \boldsymbol{\eta})=0 \quad \text { if } \quad|\hat{\boldsymbol{x}}-\boldsymbol{x}| \geq \delta,
$$

where $\delta>0$ is the so-called peridynamic horizon of interaction. Here and in the sequel, $|\cdot|$ denotes the Euclidean vector or spectral matrix norm. This cut-off in the reference configuration becomes, however, rather questionable in the presence of large deformations where it might be necessary to redefine the cut-off in the actual or present configuration.

If the material is microelastic in the sense of Silling [34] then there exists a pairwise micropotential $w$ such that $\boldsymbol{f}(\boldsymbol{x}, \hat{\boldsymbol{x}}, \boldsymbol{\eta})=\nabla_{\boldsymbol{\eta}} w(\boldsymbol{x}, \hat{\boldsymbol{x}}, \boldsymbol{\eta})$. In order to emphasise explicitly the dependence on the dimension $d$ and the horizon $\delta$, we shall use in the following the subscript $d, \delta$. One of the simplest nonlinear models that has been suggested is the proportional microelastic material model with

$$
\boldsymbol{f}_{d, \delta}(\boldsymbol{x}, \hat{\boldsymbol{x}}, \hat{\boldsymbol{u}}-\boldsymbol{u})=c_{d, \delta} s \frac{\hat{\boldsymbol{x}}+\hat{\boldsymbol{u}}-\boldsymbol{x}-\boldsymbol{u}}{|\hat{\boldsymbol{x}}+\hat{\boldsymbol{u}}-\boldsymbol{x}-\boldsymbol{u}|}, \quad s:=\frac{|\hat{\boldsymbol{x}}+\hat{\boldsymbol{u}}-\boldsymbol{x}-\boldsymbol{u}|-|\hat{\boldsymbol{x}}-\boldsymbol{x}|}{|\hat{\boldsymbol{x}}-\boldsymbol{x}|},
$$

where $s$ denotes the bond stretch that is the relative change of the length of a bond. The constant of proportionality $c_{d, \delta}$ is to be determined in such a way that the deformation energy density of a homogeneous body under isotropic expansion arising from the peridynamic model coincides with the energy density known from the classical linear elasticity theory, i.e. $\boldsymbol{\eta}=s(\hat{\boldsymbol{x}}-\boldsymbol{x})$ with some $s>0$, and

$$
\frac{1}{2} \int_{B(\boldsymbol{x} ; \delta)} w_{d, \delta}(\boldsymbol{x}, \hat{\boldsymbol{x}}, s(\hat{\boldsymbol{x}}-\boldsymbol{x})) \mathrm{d} \hat{\boldsymbol{x}} \equiv e_{\mathrm{el}, d},
$$

where

$$
e_{\mathrm{el}, 1}=\frac{9 K s^{2}}{10}, \quad e_{\mathrm{el}, 2}=\frac{12 K s^{2}}{5}, \quad e_{\mathrm{el}, 3}=\frac{9 K s^{2}}{2}
$$

is the elastic energy density from the classical linear elasticity theory for the one-, two-, and three-dimensional cases, respectively. Here, $K=E /(3(1-2 \nu))$ denotes the bulk modulus, $\nu$ the Poisson ratio, and $E$ the Young modulus. Moreover, we denote by $B(\boldsymbol{x} ; \delta) \subset \mathbb{R}^{d}$ the open ball of radius $\delta$ with respect to $|\cdot|$ and with center $\boldsymbol{x} \in \mathbb{R}^{d}$. The peridynamic model as considered so far is restricted to the Poisson ratio $\nu=1 / 4$. With $\nu=1 / 4$, one easily obtains (cf. Emmrich \& Weckner [18])

$$
c_{1, \delta}=\frac{18 K}{5 \delta^{2}}, \quad c_{2, \delta}=\frac{72 K}{5 \pi \delta^{3}}, \quad c_{3, \delta}=\frac{18 K}{\pi \delta^{4}} .
$$

We should mention here that the pairwise force function of the proportional microelastic material model fulfills the scaling property

$$
\boldsymbol{f}_{d, \varepsilon \delta}(\varepsilon \boldsymbol{x}, \varepsilon \hat{\boldsymbol{x}}, \varepsilon \boldsymbol{\eta})=\varepsilon^{-(d+1)} \boldsymbol{f}_{d, \delta}(\boldsymbol{x}, \hat{\boldsymbol{x}}, \boldsymbol{\eta}), \quad \varepsilon>0 .
$$


A first-order approximation justifies for small relative displacements $\boldsymbol{\eta}$ the general linear ansatz

$$
\boldsymbol{f}(\boldsymbol{x}, \hat{\boldsymbol{x}}, \boldsymbol{\eta})=\boldsymbol{f}_{\mathbf{0}}(\boldsymbol{x}, \hat{\boldsymbol{x}})+\boldsymbol{C}(\boldsymbol{x}, \hat{\boldsymbol{x}}) \cdot \boldsymbol{\eta}
$$

with the stiffness tensor (or micromodulus function) $\boldsymbol{C}=\boldsymbol{C}(\boldsymbol{x}, \hat{\boldsymbol{x}})$ and $\boldsymbol{f}_{\mathbf{0}}$ denoting forces in the reference configuration. Without loss of generality, we may assume $f_{0} \equiv 0$, since otherwise $f_{0}$ can be incorporated into the right-hand side $\boldsymbol{b}$. In general, the stiffness tensor $\boldsymbol{C}$ is neither definite nor dependent on the length $|\hat{\boldsymbol{x}}-\boldsymbol{x}|$ only. However, $\boldsymbol{C}$ has to be symmetric with respect to its arguments as well as with respect to its tensor structure such that $\boldsymbol{C}(\hat{\boldsymbol{x}}, \boldsymbol{x})=\boldsymbol{C}(\boldsymbol{x}, \hat{\boldsymbol{x}})$ and $\boldsymbol{C}(\boldsymbol{x}, \hat{\boldsymbol{x}})^{\top}=\boldsymbol{C}(\boldsymbol{x}, \hat{\boldsymbol{x}})$. The corresponding micropotential is then given by $w(\boldsymbol{x}, \hat{\boldsymbol{x}}, \boldsymbol{\eta})=\boldsymbol{\eta} \cdot \boldsymbol{C}(\boldsymbol{x}, \hat{\boldsymbol{x}}) \cdot \boldsymbol{\eta} / 2$. In view of (2.3), we shall require $\boldsymbol{C}(\hat{\boldsymbol{x}}, \boldsymbol{x})=0$ if $|\hat{\boldsymbol{x}}-\boldsymbol{x}| \geq \delta$.

The stiffness tensor can be shown to read as

$$
\boldsymbol{C}(\boldsymbol{x}, \hat{\boldsymbol{x}})=\lambda_{d, \delta}(|\hat{\boldsymbol{x}}-\boldsymbol{x}|)(\hat{\boldsymbol{x}}-\boldsymbol{x}) \otimes(\hat{\boldsymbol{x}}-\boldsymbol{x})
$$

for a linear microelastic material (cf. also Silling [33]). The measurable function $\lambda_{d, \delta}: \mathbb{R}_{0}^{+} \rightarrow \mathbb{R}$ with $\lambda_{d, \delta}(r)=0$ for $r \geq \delta$ determines the specific material model and depends on $d$ and $\delta$. Note that $|\boldsymbol{C}(\boldsymbol{x}, \hat{\boldsymbol{x}})|=\left|\lambda_{d, \delta}(|\hat{\boldsymbol{x}}-\boldsymbol{x}|)\right||\hat{\boldsymbol{x}}-\boldsymbol{x}|^{2}$.

The linear peridynamic equation of motion (2.1) now reads as

$$
\begin{aligned}
\rho(\boldsymbol{x}) \partial_{t}^{2} \boldsymbol{u}(\boldsymbol{x}, t) & =\int_{\mathcal{V} \cap B(\boldsymbol{x} ; \delta)} \lambda_{d, \delta}(|\hat{\boldsymbol{x}}-\boldsymbol{x}|)(\hat{\boldsymbol{x}}-\boldsymbol{x}) \otimes(\hat{\boldsymbol{x}}-\boldsymbol{x}) \cdot(\boldsymbol{u}(\hat{\boldsymbol{x}}, t)-\boldsymbol{u}(\boldsymbol{x}, t)) \mathrm{d} \hat{\boldsymbol{x}}+\boldsymbol{b}(\boldsymbol{x}, t) \\
& =:\left(L_{d, \delta} \boldsymbol{u}\right)(\boldsymbol{x}, t)+\boldsymbol{b}(\boldsymbol{x}, t), \quad(\boldsymbol{x}, t) \in \mathcal{V} \times(0, T) .
\end{aligned}
$$

A short calculation shows that $L_{d, \delta} \boldsymbol{v}$ vanishes if $\boldsymbol{v}$ is a vector field such that

$$
\boldsymbol{v}(\boldsymbol{x})=A \cdot \boldsymbol{x}+\boldsymbol{b}, \quad A^{\top}=-A,
$$

for some skew-symmetric matrix $A \in \mathbb{R}^{d \times d}$ and some vector $\boldsymbol{b} \in \mathbb{R}$. For small displacements as in the linear case, such a vector field can be seen as describing rigid body motions. In the three-dimensional case, we have $\boldsymbol{v}(\boldsymbol{x})=\boldsymbol{a} \wedge \boldsymbol{x}+\boldsymbol{b}$ for some $\boldsymbol{a}, \boldsymbol{b} \in \mathbb{R}^{3}$ (cf. also Temam \& Miranville [39, Sect. 1.4] and Oleinik et al. [28]).

For the rest of the paper, let

$$
\ell_{k, d, \delta}:=\int_{0}^{\delta} \lambda_{d, \delta}(r) r^{k+d+1} \mathrm{~d} r, \quad k=0,1,2, \ldots, \quad \ell_{d, \delta}^{|\cdot|}:=\int_{0}^{\delta}\left|\lambda_{d, \delta}(r)\right| r^{d+1} \mathrm{~d} r .
$$

By virtue of (2.5), we require

$$
\frac{s^{2}}{4} \int_{B(0 ; \delta)} \boldsymbol{\xi} \cdot \lambda_{d, \delta}(|\boldsymbol{\xi}|) \boldsymbol{\xi} \otimes \boldsymbol{\xi} \cdot \boldsymbol{\xi} \mathrm{d} \boldsymbol{\xi}=e_{\mathrm{el}, d}
$$

which yields a first assumption on $\lambda_{d, \delta}$, namely,

$$
\ell_{2, d, \delta}=\frac{4 e_{\mathrm{el}, d}}{s^{2} \sigma_{d}}, \quad \text { i.e., } \ell_{2,1, \delta}=\frac{9 K}{5}, \quad \ell_{2,2, \delta}=\frac{24 K}{5 \pi}, \quad \ell_{2,3, \delta}=\frac{9 K}{2 \pi},
$$

where $\sigma_{d}$ comes from the integration with respect to the spherical angle coordinates such that $\sigma_{1}=2, \sigma_{2}=2 \pi, \sigma_{3}=4 \pi$. 
Well-posedness of the linear peridynamic equation of motion has been studied by the authors in [43] for the unbounded one-dimensional case and in [18] for the bounded two- and three-dimensional case in a framework with $L^{p}(\mathcal{V})^{d}$ with sufficiently large $p$. The proofs of the two statements below follow essentially the same lines as in [18] and shall be omitted here.

Proposition 2.1. If $\ell_{d, \delta}^{|\cdot|}<\infty$ then the integral operator $L_{d, \delta}$ defined by (2.8) is a linear mapping in $L^{\infty}(\mathcal{V})^{d}$ with

$$
\left\|L_{d, \delta}\right\|_{\mathcal{L}\left(L^{\infty}(\mathcal{V})^{d}\right)} \leq 2 \ell_{d, \delta}^{|\cdot|} \sigma_{d}
$$

TheOREM 2.2. If $\ell_{d, \delta}^{|\cdot|}<\infty$ then there exists for every $\boldsymbol{u}_{0}, \dot{\boldsymbol{u}}_{0} \in L^{\infty}(\mathcal{V})^{d}, \quad \boldsymbol{b} \in$ $L^{1}\left(0, T ; L^{\infty}(\mathcal{V})^{d}\right)$ a unique mild solution $\boldsymbol{u} \in \mathcal{C}^{1}\left([0, T] ; L^{\infty}(\mathcal{V})^{d}\right)$ to the initial-value problem (2.8), (2.2) that satisfies the a priori estimate

$$
\|\boldsymbol{u}\|_{\mathcal{C}^{1}\left([0, T] ; L^{\infty}(\mathcal{V})^{d}\right)} \leq C_{d, \delta}\left(\left\|\boldsymbol{u}_{0}\right\|_{L^{\infty}(\mathcal{V})^{d}}+\left\|\dot{\boldsymbol{u}}_{0}\right\|_{L^{\infty}(\mathcal{V})^{d}}+\|\boldsymbol{b}\|_{L^{1}\left(0, T ; L^{\infty}(\mathcal{V})^{d}\right)}\right)
$$

with

$$
C_{d, \delta}=\max \left(1, T, 2 T \ell_{d, \delta}^{|\cdot|} \sigma_{d}\right) \cosh \left(T \sqrt{2 \ell_{d, \delta}^{|\cdot|} \sigma_{d}}\right) .
$$

If $\boldsymbol{b} \in \mathcal{C}\left([0, T] ; L^{\infty}(\mathcal{V})^{d}\right)$ then $\boldsymbol{u} \in \mathcal{C}^{2}\left([0, T] ; L^{\infty}(\mathcal{V})^{d}\right)$.

The stability estimate (2.12) with (2.13) immediately follows from the representation of the solution by means of Duhamel's principle (cf. again [18]),

$$
\begin{aligned}
\boldsymbol{u}(t)= & \cos \left(t \sqrt{-L_{d, \delta}}\right) \boldsymbol{u}_{0}+{\sqrt{-L_{d, \delta}}-1}^{-1} \sin \left(t \sqrt{-L_{d, \delta}}\right) \dot{\boldsymbol{u}}_{0} \\
& +\int_{0}^{t}{\sqrt{-L_{d, \delta}}}^{-1} \sin \left((t-s) \sqrt{-L_{d, \delta}}\right) \boldsymbol{b}(s) \mathrm{d} s
\end{aligned}
$$

with the formal notation

$$
\cos \left(t \sqrt{-L_{d, \delta}}\right):=\sum_{n=0}^{\infty} \frac{t^{2 n}}{(2 n) !} L_{d, \delta}^{n}, \quad{\sqrt{-L_{d, \delta}}}^{-1} \sin \left(t \sqrt{-L_{d, \delta}}\right):=\sum_{n=0}^{\infty} \frac{t^{2 n+1}}{(2 n+1) !} L_{d, \delta}^{n} .
$$

The series are uniformly convergent on any compact time interval.

It should be noted that, due to (2.10), the quantities $\ell_{2, d, \delta}$ are independent of $\delta$ and thus, because of (2.11) and

$$
0<\ell_{2, d, \delta} \leq \delta^{2} \ell_{d, \delta}^{|\cdot|}
$$

the family of operators $L_{d, \delta}: L^{\infty}(\mathcal{V})^{d} \rightarrow L^{\infty}(\mathcal{V})^{d}$ may not be uniformly bounded as $\delta \rightarrow 0$. Reasoning in the same way shows that also the stability constant $C_{d, \delta}$ from (2.13) behaves badly as $\delta \rightarrow 0$. Furthermore, we can prove the following results.

Proposition 2.3. If $\ell_{d, \delta}^{|\cdot|}<\infty$ then the integral operator $L_{d, \delta}$ defined by (2.8) is a linear mapping in $L^{2}(\mathcal{V})^{d}$ with

$$
\left\|L_{d, \delta}\right\|_{\mathcal{L}\left(L^{2}(\mathcal{V})^{d}\right)} \leq 2 \ell_{d, \delta}^{|\cdot|} \sigma_{d} .
$$


Moreover, $L_{d, \delta}: L^{2}(\mathcal{V})^{d} \rightarrow L^{2}(\mathcal{V})^{d}$ is self-adjoint, i.e.,

$$
\left(L_{d, \delta} \boldsymbol{v}, \boldsymbol{w}\right)_{L^{2}(\mathcal{V})^{d}}=\left(\boldsymbol{v}, L_{d, \delta} \boldsymbol{w}\right)_{L^{2}(\mathcal{V})^{d}} \quad \forall \boldsymbol{v}, \boldsymbol{w} \in L^{2}(\mathcal{V})^{d} .
$$

If $\lambda_{d, \delta}$ is nonnegative then $L_{d, \delta}: L^{2}(\mathcal{V})^{d} \rightarrow L^{2}(\mathcal{V})^{d}$ is also dissipative, i.e.

$$
\left(L_{d, \delta} \boldsymbol{v}, \boldsymbol{v}\right)_{L^{2}(\mathcal{V})^{d}} \leq 0 \quad \forall \boldsymbol{v} \in L^{2}(\mathcal{V})^{d},
$$

where $\left(L_{d, \delta} \boldsymbol{v}, \boldsymbol{v}\right)_{L^{2}(\mathcal{V})^{d}}=0$ if and only if $\boldsymbol{v}$ is of type (2.9) or $\lambda_{d, \delta}$ vanishes identically.

Proof. We immediately find

$$
\left|\left(L_{d, \delta} \boldsymbol{v}\right)(\boldsymbol{x})\right| \leq \int_{\mathcal{V}}\left|\lambda_{d, \delta}(|\hat{\boldsymbol{x}}-\boldsymbol{x}|)\right||\hat{\boldsymbol{x}}-\boldsymbol{x}|^{2}|\boldsymbol{v}(\hat{\boldsymbol{x}})| \mathrm{d} \hat{\boldsymbol{x}}+\ell_{d, \delta}^{|\cdot|} \sigma_{d}|\boldsymbol{v}(x)| .
$$

Denoting the integral on the right-hand side by $I(\boldsymbol{x})$, an application of the CauchySchwarz inequality yields

$$
|I(\boldsymbol{x})|^{2} \leq \ell_{d, \delta}^{|\cdot|} \sigma_{d} \int_{\mathcal{V}}\left|\lambda_{d, \delta}(|\hat{\boldsymbol{x}}-\boldsymbol{x}|)\right||\hat{\boldsymbol{x}}-\boldsymbol{x}|^{2}|\boldsymbol{v}(\hat{\boldsymbol{x}})|^{2} \mathrm{~d} \hat{\boldsymbol{x}}
$$

Integration then gives

$$
\int_{\mathcal{V}}|I(\boldsymbol{x})|^{2} \mathrm{~d} \boldsymbol{x} \leq \ell_{d, \delta}^{|\cdot|} \sigma_{d} \int_{\mathcal{V}}\left(\int_{\mathcal{V}}\left|\lambda_{d, \delta}(|\hat{\boldsymbol{x}}-\boldsymbol{x}|)\right||\hat{\boldsymbol{x}}-\boldsymbol{x}|^{2} \mathrm{~d} \boldsymbol{x}\right)|\boldsymbol{v}(\hat{\boldsymbol{x}})|^{2} \mathrm{~d} \hat{\boldsymbol{x}} \leq\left(\ell_{d, \delta}^{|\cdot|} \sigma_{d}\right)^{2}\|v\|_{L^{2}(\mathcal{V})^{d}}^{2},
$$

from which the first assertion follows.

A straightforward calculation shows

$$
\begin{gathered}
\left(L_{d, \delta} \boldsymbol{v}, \boldsymbol{w}\right)_{L^{2}(\mathcal{V})^{d}}=-\frac{1}{2} \iint_{\mathcal{V} \mathcal{V}} \lambda_{d, \delta}(|\hat{\boldsymbol{x}}-\boldsymbol{x}|)((\boldsymbol{v}(\hat{\boldsymbol{x}})-\boldsymbol{v}(\boldsymbol{x})) \cdot(\hat{\boldsymbol{x}}-\boldsymbol{x})) \times \\
((\boldsymbol{w}(\hat{\boldsymbol{x}})-\boldsymbol{w}(\boldsymbol{x})) \cdot(\hat{\boldsymbol{x}}-\boldsymbol{x})) \mathrm{d} \hat{\boldsymbol{x}} \mathrm{d} \boldsymbol{x}
\end{gathered}
$$

which symmetry and thus self-adjointness of $L_{d, \delta}: L^{2}(\mathcal{V})^{d} \rightarrow L^{2}(\mathcal{V})^{d}$ implies. In the case $\boldsymbol{v}=\boldsymbol{w}$, this also proves dissipativity if $\lambda_{d, \delta}$ is nonnegative. Applying [39, Proposition 1.2] shows that $(\boldsymbol{v}(\hat{\boldsymbol{x}})-\boldsymbol{v}(\boldsymbol{x})) \cdot(\hat{\boldsymbol{x}}-\boldsymbol{x})$ vanishes identically if and only if $\boldsymbol{v}$ is of type (2.9). This proves the last assertion.

By standard arguments for abstract differential equations with a linear bounded operator and Duhamel's principle (cf. e.g. Emmrich [19, Ch. 7]), we can prove the following theorem.

TheOREM 2.4. If $\ell_{d, \delta}^{|\cdot|}<\infty$ then there exists for every $\boldsymbol{u}_{0}, \dot{\boldsymbol{u}}_{0} \in L^{2}(\mathcal{V})^{d}, \quad \boldsymbol{b} \in$ $L^{1}\left(0, T ; L^{2}(\mathcal{V})^{d}\right)$ a unique mild solution $\boldsymbol{u} \in \mathcal{C}^{1}\left([0, T] ; L^{2}(\mathcal{V})^{d}\right)$ to the initial-value problem (2.8), (2.2) that satisfies the a priori estimate (2.12), (2.13) with $L^{\infty}(\mathcal{V})^{d}$ being replaced by $L^{2}(\mathcal{V})^{d}$. If $\boldsymbol{b} \in \mathcal{C}\left([0, T] ; L^{2}(\mathcal{V})^{d}\right)$ then $\boldsymbol{u} \in \mathcal{C}^{2}\left([0, T] ; L^{2}(\mathcal{V})^{d}\right)$.

The solution is again given by $(2.14), L_{d, \delta}: L^{2}(\mathcal{V})^{d} \rightarrow L^{2}(\mathcal{V})^{d}$ is the generator of a strongly continuous cosine function on $L^{2}(\mathcal{V})^{d}$, and the operator describing the corresponding one-dimensional system is the generator of a strongly continuous semigroup on $L^{2}(\mathcal{V})^{d} \times L^{2}(\mathcal{V})^{d}$ (cf. also Arendt et al. [3, Theorem 3.14.7, Corolarry 3.14.9] and Fattorini [21]). 
As in Proposition 2.1 and Theorem 2.2, we cannot conclude uniform boundedness with respect to $\delta$. This problem can be overcome as follows. As usual, we denote by $|\cdot|_{H^{1}(\mathcal{V})^{d}}$ the standard semi-norm of $H^{1}(\mathcal{V})^{d}$.

Lemma 2.1. Assume $v \in H^{1}(\mathcal{V})$ and let $\phi \in L^{1}\left(\mathbb{R}^{d}\right), \phi \geq 0$. Then

$$
\iint_{\mathcal{V}} \phi(\hat{\boldsymbol{x}}-\boldsymbol{x}) \frac{|v(\hat{\boldsymbol{x}})-v(\boldsymbol{x})|^{2}}{|\hat{\boldsymbol{x}}-\boldsymbol{x}|^{2}} \mathrm{~d} \hat{\boldsymbol{x}} \mathrm{d} \boldsymbol{x} \leq C\|\phi\|_{L^{1}\left(\mathbb{R}^{d}\right)}|v|_{H^{1}(\mathcal{V})}^{2},
$$

where $C$ depends only on $\mathcal{V}$.

The lemma is a special case of Bourgain et al. [11, Theorem 1].

Proposition 2.5. Let $\ell_{d, \delta}^{|\cdot|}<\infty$. If $\lambda_{d, \delta}$ is nonnegative and fulfills (2.10) then $L_{d, \delta}$ is a linear mapping on $H^{1}(\mathcal{V})^{d}$ with

$$
\left|\left(L_{d, \delta} \boldsymbol{v}, \boldsymbol{w}\right)_{L^{2}(\mathcal{V})^{d}}\right| \leq C \ell_{2, d, \delta}|\boldsymbol{v}|_{H^{1}(\mathcal{V})^{d}}|\boldsymbol{w}|_{H^{1}(\mathcal{V})^{d}} \quad \forall \boldsymbol{v}, \boldsymbol{w} \in H^{1}(\mathcal{V})^{d},
$$

where $C$ depends on the domain and its dimension but is independent of $\delta$.

Proof. Let $\phi(\boldsymbol{\xi}):=\lambda_{d, \delta}(|\boldsymbol{\xi}|)|\boldsymbol{\xi}|^{4} \quad\left(\boldsymbol{\xi} \in \mathbb{R}^{d}\right)$. From (2.17), we conclude with the Cauchy-Schwarz inequality

$$
\begin{aligned}
& \left|\left(L_{d, \delta} \boldsymbol{v}, \boldsymbol{w}\right)_{L^{2}(\mathcal{V})^{d}}\right| \leq \\
& \frac{1}{2}\left(\int_{\mathcal{V} \mathcal{V}} \phi(\hat{\boldsymbol{x}}-\boldsymbol{x}) \frac{|\boldsymbol{v}(\hat{\boldsymbol{x}})-\boldsymbol{v}(\boldsymbol{x})|^{2}}{|\hat{\boldsymbol{x}}-\boldsymbol{x}|^{2}} \mathrm{~d} \hat{\boldsymbol{x}} \mathrm{d} \boldsymbol{x}\right)^{1 / 2}\left(\iint_{\mathcal{V} \mathcal{V}} \phi(\hat{\boldsymbol{x}}-\boldsymbol{x}) \frac{|\boldsymbol{w}(\hat{\boldsymbol{x}})-\boldsymbol{w}(\boldsymbol{x})|^{2}}{|\hat{\boldsymbol{x}}-\boldsymbol{x}|^{2}} \mathrm{~d} \hat{\boldsymbol{x}} \mathrm{d} \boldsymbol{x}\right)^{1 / 2} .
\end{aligned}
$$

Lemma 2.1 due to Bourgain et al. [11] yields

$$
\left|\left(L_{d, \delta} \boldsymbol{v}, \boldsymbol{w}\right)_{L^{2}(\mathcal{V})^{d}}\right| \leq C\|\phi\|_{L^{1}\left(\mathbb{R}^{d}\right)}|\boldsymbol{v}|_{H^{1}(\mathcal{V})^{d}}|\boldsymbol{w}|_{H^{1}(\mathcal{V})^{d}}
$$

with $C$ only depending on $\mathcal{V}$ and $d$. The assertion now follows in view of

$$
\|\phi\|_{L^{1}\left(\mathbb{R}^{d}\right)}=\int_{B(0 ; \delta)} \lambda_{d, \delta}(|\boldsymbol{\xi}|)|\boldsymbol{\xi}|^{4} \mathrm{~d} \boldsymbol{\xi}=\ell_{2, d, \delta} \sigma_{d} .
$$

The proposition above shows in particular that

$$
\left\|L_{d, \delta}\right\|_{\mathcal{L}\left(H^{1}(\mathcal{V})^{d},\left(H^{1}(\mathcal{V})^{d}\right)^{*}\right)} \leq C \ell_{2, d, \delta}
$$

Because of (2.10), the family of linear bounded operators $L_{d, \delta}: H^{1}(\mathcal{V})^{d} \rightarrow\left(H^{1}(\mathcal{V})^{d}\right)^{*}$ is thus uniformly bounded with respect to $\delta$.

As we can infer from (2.17) and the proof above, $L_{d, \delta}$ might also be considered as a linear bounded operator from $W^{1, \infty}(\mathcal{V})^{d}$ in its dual with $\left\|L_{d, \delta}\right\|_{\mathcal{L}_{\left(W^{1, \infty}(\mathcal{V})^{d},\left(W^{1, \infty}(\mathcal{V})^{d}\right)^{*}\right)}}$ being bounded independently of $\delta$.

An immediate consequence of the preceding propositions is the following theorem.

THEOREM 2.6. Let $\ell_{d, \delta}^{|\cdot|}<\infty$ and assume that $\lambda_{d, \delta}$ is nonnegative and fulfills (2.10). The unique solution $\boldsymbol{u} \in \mathcal{C}^{2}\left([0, T] ; L^{2}(\mathcal{V})^{d}\right)$ to the initial-value problem (2.8), (2.2) with $\boldsymbol{u}_{0} \in H^{1}(\mathcal{V})^{d}, \dot{\boldsymbol{u}}_{0} \in L^{2}(\mathcal{V})^{d}$, and $\boldsymbol{b} \in \mathcal{C}\left([0, T] ; L^{2}(\mathcal{V})^{d}\right)$ then satisfies the a priori estimate

$$
\|\boldsymbol{u}\|_{\mathcal{C}^{1}\left([0, T] ; L^{2}(\mathcal{V})^{d}\right)} \leq C\left(\left\|\boldsymbol{u}_{0}\right\|_{H^{1}(\mathcal{V})^{d}}+\left\|\dot{\boldsymbol{u}}_{0}\right\|_{L^{2}(\mathcal{V})^{d}}+\|\boldsymbol{b}\|_{L^{2}\left(0, T ; L^{2}(\mathcal{V})^{d}\right)}\right)
$$


with $C$ being independent of $\delta$.

Proof. According to Theorem 2.4, there is a unique solution $\boldsymbol{u} \in \mathcal{C}^{2}\left([0, T] ; L^{2}(\mathcal{V})^{d}\right)$. For proving (2.18), we firstly observe that for all $\boldsymbol{v} \in \mathcal{C}^{1}\left([0, T] ; L^{2}(\mathcal{V})^{d}\right)$

$$
\frac{1}{2} \frac{\mathrm{d}}{\mathrm{d} t}(\boldsymbol{v}(t), \boldsymbol{v}(t))_{L^{2}(\mathcal{V})^{d}}=\left(\partial_{t} \boldsymbol{v}(t), \boldsymbol{v}(t)\right)_{L^{2}(\mathcal{V})^{d}} ;
$$

an analogous relation holds true for the second time derivative if $\boldsymbol{v} \in \mathcal{C}^{2}\left([0, T] ; L^{2}(\mathcal{V})^{d}\right)$. Testing (2.8) by $\partial_{t} \boldsymbol{u}$ then leads (with the symmetry of $L_{d, \delta}$ ) to

$$
\begin{aligned}
\frac{\rho}{2} \frac{\mathrm{d}}{\mathrm{d} t}\left\|\partial_{t} \boldsymbol{u}(t)\right\|_{L^{2}(\mathcal{V})^{d}}^{2} & =\left(L_{d, \delta} \boldsymbol{u}(t), \partial_{t} \boldsymbol{u}(t)\right)_{L^{2}(\mathcal{V})^{d}}+\left(\boldsymbol{b}(t), \partial_{t} \boldsymbol{u}(t)\right)_{L^{2}(\mathcal{V})^{d}} \\
& \leq \frac{1}{2} \frac{\mathrm{d}}{\mathrm{d} t}\left(L_{d, \delta} \boldsymbol{u}(t), \boldsymbol{u}(t)\right)_{L^{2}(\mathcal{V})^{d}}+\|\boldsymbol{b}(t)\|_{L^{2}(\mathcal{V})^{d}}\left\|\partial_{t} \boldsymbol{u}(t)\right\|_{L^{2}(\mathcal{V})^{d}}
\end{aligned}
$$

With Young's inequality, integration, dissipativity, and Gronwall's lemma, we come up with

$$
\left\|\partial_{t} \boldsymbol{u}(t)\right\|_{L^{2}(\mathcal{V})^{d}}^{2} \leq C\left(-\left(L_{d, \delta} \boldsymbol{u}_{0}, \boldsymbol{u}_{0}\right)_{L^{2}(\mathcal{V})^{d}}+\left\|\partial_{t} \boldsymbol{u}_{0}\right\|_{L^{2}(\mathcal{V})^{d}}^{2}+\int_{0}^{t}\|\boldsymbol{b}(s)\|_{L^{2}(\mathcal{V})^{d}}^{2} \mathrm{~d} s\right),
$$

where $C$ depends on $1 / \rho \in L^{\infty}(\mathcal{V})$ and $T$ only. The assertion follows with Proposition 2.5 and since

$$
\|\boldsymbol{u}(t)\|_{L^{2}(\mathcal{V})^{d}} \leq\left\|\boldsymbol{u}_{0}\right\|_{L^{2}(\mathcal{V})^{d}}+\int_{0}^{t}\left\|\partial_{t} \boldsymbol{u}(s)\right\|_{L^{2}(\mathcal{V})^{d}} \mathrm{~d} s .
$$

A main advantage of (2.18) is the fact that the stability constant now is independent of $\delta$ and thus furnishes uniform boundedness of a sequence of solutions $\boldsymbol{u}_{\delta}$ as $\delta \rightarrow 0$.

So far, our existence results were based upon abstract differential equations governed by a linear bounded operator that maps a Banach space into itself. However, by virtue of Proposition 2.5 in combination with Proposition 2.3, we may also think of applying the theory of weak solutions to second-order evolution equations based upon the Gelfand triple $H^{1}(\mathcal{V})^{d} \subset L^{2}(\mathcal{V})^{d} \subset\left(H^{1}(\mathcal{V})^{d}\right)^{*}$ (cf. e.g. Wloka [44, Ch. V]). This would allow to consider the peridynamic equation of motion (2.8) in the same functional analytic setting as the Navier equation of linear elasticity theory. Unfortunately, the operator $-L_{d, \delta}: H^{1}(\mathcal{V})^{d} \rightarrow\left(H^{1}(\mathcal{V})^{d}\right)^{*}$ has not yet been proven to satisfy a Gårding inequality.

\section{The limit behaviour for vanishing non-locality}

In the following, we consider $\delta \in \Lambda$ where $\Lambda \subset \mathbb{R}^{+}$is a null sequence with $\max \Lambda<\delta_{0}$ for some $\delta_{0}>0$. We set

$$
\mathcal{V}_{0}:=\left\{\boldsymbol{z} \in \mathcal{V}: \operatorname{dist}(\boldsymbol{z}, \partial \mathcal{V})>\delta_{0}\right\}
$$

Corollary 3.1. Let $\ell_{d, \delta}^{|\cdot|}<\infty$ and assume that $\lambda_{d, \delta}$ is nonnegative and fulfills (2.10). The sequence $\left\{\boldsymbol{u}_{\delta}\right\}_{\delta \in \Lambda}$ of solutions to the initial-value problem (2.8), (2.2) with $\boldsymbol{u}_{0} \in$ 
$H^{1}(\mathcal{V})^{d}, \dot{\boldsymbol{u}}_{0} \in L^{2}(\mathcal{V})^{d}, \boldsymbol{b} \in \mathcal{C}\left([0, T] ; L^{2}(\mathcal{V})^{d}\right)$ and the sequence of its time derivatives then possesses a subsequence that converges weakly* in $L^{\infty}\left(0, T ; L^{2}(\mathcal{V})^{d}\right)$ as $\delta \rightarrow 0(\delta \in \Lambda)$.

Proof. The result is a direct consequence of Theorem 2.6 applying the BanachAlaoglu theorem (upon noting that $L^{\infty}\left(0, T ; L^{2}(\mathcal{V})^{d}\right)$ is the dual of the separable Banach space $\left.L^{1}\left(0, T ; L^{2}(\mathcal{V})^{d}\right)\right)$.

It remains open for future work to study the properties of the weak* limit of $\left\{\boldsymbol{u}_{\delta}\right\}_{\delta \in \Lambda}$. Also the following observations give rise to further study.

The classical Navier equation of linear elasticity reads for $(\boldsymbol{x}, t) \in \mathcal{V} \times(0, T)$ as

$$
\rho(\boldsymbol{x}) \partial_{t}^{2} \boldsymbol{u}(\boldsymbol{x}, t)=\mu \Delta \boldsymbol{u}(\boldsymbol{x}, t)+(\lambda+\mu) \operatorname{grad} \operatorname{div} \boldsymbol{u}(\boldsymbol{x}, t)+\boldsymbol{b}(\boldsymbol{x}, t)=:(L \boldsymbol{u})(\boldsymbol{x}, t)+\boldsymbol{b}(\boldsymbol{x}, t)
$$

with the Lamé coefficients $\mu=E /(2(1+\nu))$ and $\lambda=\nu E /((1-2 \nu)(1+\nu))$, cf. e.g. Temam \& Miranville [39]. Note that for the Poisson ratio $\nu=1 / 4$, we find $\mu=\lambda=$ $2 E / 5=3 K / 5$. The Navier equation is supplemented by initial conditions (2.2) and appropriate boundary conditions.

THEOREM 3.2. Let $\ell_{d, \delta}^{|\cdot|}<\infty$ for all $\delta \in \Lambda$ and assume (2.10). If $\boldsymbol{v} \in \mathcal{C}^{2}(\mathcal{V})^{d}$ and $\lambda_{d, \delta}$ is nonnegative, then

$$
\left\|L_{d, \delta} \boldsymbol{v}-L \boldsymbol{v}\right\|_{L^{\infty}\left(\mathcal{V}_{0}\right)^{d}} \rightarrow 0 \quad \text { as } \quad \delta \rightarrow 0 \quad(\delta \in \Lambda) .
$$

If $\boldsymbol{v} \in \mathcal{C}^{m}(\mathcal{V})^{d}(m \geq 2)$ then the expansion

$$
\left(L_{d, \delta} \boldsymbol{v}\right)(\boldsymbol{x})=(L \boldsymbol{v})(\boldsymbol{x})+\sum_{n=2}^{[m / 2]}\left(L_{d, \delta}^{(2 n)} \boldsymbol{v}\right)(\boldsymbol{x})+\boldsymbol{R}_{m ; d, \delta}(\boldsymbol{v} ; \boldsymbol{x})
$$

holds for all $\boldsymbol{x} \in \mathcal{V}_{0}$ and $\delta \in \Lambda$. Here, $L_{d, \delta}^{(2 n)}$ is a differential operator of order $2 n$ given by (3.3), (3.4), and (3.5), respectively, and the remainder satisfies $\left\|\boldsymbol{R}_{m ; d, \delta}(\boldsymbol{v} ; \boldsymbol{x})\right\|_{L^{\infty}\left(\mathcal{V}_{0}\right)^{d}}=o\left(\delta^{m} \ell_{d, \delta}^{|\cdot|}\right)$ with $\ell_{d, \delta}^{|\cdot|} \geq C \delta^{-2}$. If, in addition, $\lambda_{d, \delta}$ is nonnegative then

$$
\left\|L_{d, \delta}^{(2 n)} \boldsymbol{v}\right\|_{L^{\infty}\left(\mathcal{V}_{0}\right)^{d}} \leq C \delta^{2(n-1)} \ell_{2, d, \delta}\|\boldsymbol{v}\|_{\mathcal{C}^{2 n}\left(\overline{\mathcal{V}_{0}}\right)^{d}}, \quad\left\|\boldsymbol{R}_{m ; d, \delta}(\boldsymbol{v} ; \boldsymbol{x})\right\|_{L^{\infty}\left(\mathcal{V}_{0}\right)^{d}}=o\left(\delta^{m-2}\right)
$$

with $\ell_{2, d, \delta}$ being independent of $\delta$ and given by (2.10).

Proof. For $\boldsymbol{x} \in \overline{\mathcal{V}_{0}}$, the closed ball $\bar{B}(\boldsymbol{x} ; \delta) \ni \hat{\boldsymbol{x}}$ lies in $\mathcal{V}$ for all $\delta \in \Lambda$. The smoothness assumed for $\boldsymbol{v}$ then allows the Taylor expansion

$$
\boldsymbol{v}(\hat{\boldsymbol{x}})-\boldsymbol{v}(\boldsymbol{x})=\sum_{k=1}^{m} \frac{1}{k !}((\hat{\boldsymbol{x}}-\boldsymbol{x}) \cdot \nabla)^{k} \boldsymbol{v}(\boldsymbol{x})+\boldsymbol{r}_{m}(\boldsymbol{v} ; \boldsymbol{x}, \hat{\boldsymbol{x}})
$$

with the remainder

$$
\boldsymbol{r}_{m}(\boldsymbol{v} ; \boldsymbol{x}, \hat{\boldsymbol{x}})=\int_{0}^{1} \frac{(1-\theta)^{m-1}}{((m-1) !}((\hat{\boldsymbol{x}}-\boldsymbol{x}) \cdot \nabla)^{m}(\boldsymbol{v}(\boldsymbol{x}+\theta(\hat{\boldsymbol{x}}-\boldsymbol{x}))-\boldsymbol{v}(\boldsymbol{x})) \mathrm{d} \theta=o\left(|\hat{\boldsymbol{x}}-\boldsymbol{x}|^{m}\right) .
$$

Inserting the Taylor expansion into the definition of $L_{d, \delta}$ (see (2.8)) yields

$$
\left(L_{d, \delta} \boldsymbol{v}\right)(\boldsymbol{x})=\sum_{k=1}^{m}\left(L_{d, \delta}^{(k)} \boldsymbol{v}\right)(\boldsymbol{x})+\boldsymbol{R}_{m ; d, \delta}(\boldsymbol{v} ; \boldsymbol{x}),
$$


where

$$
\left(L_{d, \delta}^{(k)} \boldsymbol{v}\right)(\boldsymbol{x}):=\frac{1}{k !} \int_{B(\boldsymbol{x} ; \delta)} \lambda_{d, \delta}(|\hat{\boldsymbol{x}}-\boldsymbol{x}|)(\hat{\boldsymbol{x}}-\boldsymbol{x}) \otimes(\hat{\boldsymbol{x}}-\boldsymbol{x})((\hat{\boldsymbol{x}}-\boldsymbol{x}) \cdot \nabla)^{k} \boldsymbol{v}(\boldsymbol{x}) \mathrm{d} \hat{\boldsymbol{x}}
$$

and

$$
\boldsymbol{R}_{m ; d, \delta}(\boldsymbol{v} ; \boldsymbol{x})=\int_{B(\boldsymbol{x} ; \delta)} \lambda_{d, \delta}(|\hat{\boldsymbol{x}}-\boldsymbol{x}|)(\hat{\boldsymbol{x}}-\boldsymbol{x}) \otimes(\hat{\boldsymbol{x}}-\boldsymbol{x}) \boldsymbol{r}_{m}(\boldsymbol{v} ; \boldsymbol{x}, \hat{\boldsymbol{x}}) \mathrm{d} \hat{\boldsymbol{x}} .
$$

It follows $\left(L_{d, \delta}^{(k)} \boldsymbol{v}\right)(\boldsymbol{x})=0$ if $k$ is odd since then the integrand is an odd function in $\hat{\boldsymbol{x}}-\boldsymbol{x}$.

For the one-dimensional case, we immediately find

$$
\left(L_{1, \delta}^{(k)} v\right)(x)= \begin{cases}0 & \text { if } k \text { is odd } \\ \frac{2}{k !} \ell_{k, 1, \delta} v^{(k)}(x) & \text { if } k \text { is even }\end{cases}
$$

In particular, (2.10) furnishes $\left(L_{1, \delta}^{(2)} v\right)(x)=9 K v^{\prime \prime}(x) / 5=(L v)(x)$.

Introducing spherical coordinates, we obtain for the two-dimensional case

$\left(L_{2, \delta}^{(k)} \boldsymbol{v}\right)(\boldsymbol{x})=\ell_{k, 2, \delta} \sum_{j=0}^{k} \frac{1}{j !(k-j) !} \int_{0}^{2 \pi}\left(\begin{array}{cc}\cos ^{2} \phi & \cos \phi \sin \phi \\ \cos \phi \sin \phi & \sin ^{2} \phi\end{array}\right) \cos ^{k-j} \phi \sin ^{j} \phi \mathrm{d} \phi \partial^{(k-j, j)} \boldsymbol{v}(\boldsymbol{x})$

and for the three-dimensional case

$$
\begin{aligned}
& \quad\left(L_{3, \delta}^{(k)} \boldsymbol{v}\right)(\boldsymbol{x}) \\
& =\ell_{k, 3, \delta} \sum_{j_{1}+j_{2}+j_{3}=k} \frac{1}{j_{1} ! j_{2} ! j_{3} !} \int_{0}^{2 \pi} \int_{-\pi / 2}^{\pi / 2}\left(\begin{array}{ccc}
\cos ^{2} \phi \cos ^{2} \psi & \cos \phi \sin \phi \cos ^{2} \psi & \cos \phi \cos \psi \sin \psi \\
\cos \phi \sin \phi \cos ^{2} \psi & \sin ^{2} \phi \cos ^{2} \psi & \sin \phi \cos \psi \sin \psi \\
\cos \phi \cos \psi \sin \psi & \sin \phi \cos \psi \sin \psi & \sin ^{2} \psi
\end{array}\right) \\
& \quad \times(\cos \phi \cos \psi)^{j_{1}}(\sin \phi \cos \psi)^{j_{2}} \sin ^{j_{3}} \psi \cos \psi \mathrm{d} \psi \mathrm{d} \phi \partial^{\left(j_{1}, j_{2}, j_{3}\right)} \boldsymbol{v}(\boldsymbol{x}) .
\end{aligned}
$$

Here, we write spatial derivatives using multiindices, $\partial^{\alpha} \equiv \partial_{x_{1}}^{\alpha_{1}} \cdots \partial_{x_{d}}^{\alpha_{d}}$ for $\alpha=$ $\left(\alpha_{1}, \ldots, \alpha_{d}\right)$. With $x_{1}, \ldots, x_{d}$ and $v_{1}, \ldots, v_{d}$, the components of $\boldsymbol{x}$ and $\boldsymbol{v}$, respectively, are denoted.

In particular, we find with $(2.10)$

$$
\left(L_{2, \delta}^{(2)} \boldsymbol{v}\right)(\boldsymbol{x})=\frac{3 K}{5}\left(\begin{array}{c}
3 \partial^{(20)} v_{1}+2 \partial^{(11)} v_{2}+\partial^{(02)} v_{1} \\
\partial^{(20)} v_{2}+2 \partial^{(11)} v_{1}+3 \partial^{(02)} v_{2}
\end{array}\right)(\boldsymbol{x})=(L \boldsymbol{v})(\boldsymbol{x})
$$

as well as

$$
\begin{aligned}
& \left(L_{3, \delta}^{(2)} \boldsymbol{v}\right)(\boldsymbol{x}) \\
& =\frac{3 K}{5}\left(\begin{array}{cc}
3 \partial^{(200)} v_{1}+2 \partial^{(110)} v_{2}+2 \partial^{(101)} v_{3}+\partial^{(020)} v_{1}+ & \partial^{(002)} v_{1} \\
\partial^{(200)} v_{2}+2 \partial^{(110)} v_{1}+ & 3 \partial^{(020)} v_{2}+2 \partial^{(011)} v_{3}+\partial^{(002)} v_{2} \\
\partial^{(200)} v_{3}+ & 2 \partial^{(101)} v_{1}+\partial^{(020)} v_{3}+2 \partial^{(011)} v_{2}+3 \partial^{(002)} v_{3}
\end{array}\right)(\boldsymbol{x}) \\
& =(L \boldsymbol{v})(\boldsymbol{x}) \text {. }
\end{aligned}
$$


This, together with $\boldsymbol{v} \in \mathcal{C}^{m}(\mathcal{V})^{d}$ and (2.15), proves the expansion asserted. Moreover, for nonnegative $\lambda_{d, \delta}$, we observe that

$$
0 \leq \ell_{k, d, \delta} \leq \delta^{k-2} \ell_{2, d, \delta}, \quad k=2,3, \ldots, m .
$$

The uniform convergence (3.2) is a direct consequence of the preceding results with $m=2$ since the second-order derivatives of $\boldsymbol{v} \in \mathcal{C}^{2}(\mathcal{V})^{d}$ are uniformly continuous on $\bigcup_{\delta \in \Lambda} \bigcup_{\boldsymbol{x} \in \overline{\mathcal{V}_{0}}} \bar{B}(\boldsymbol{x} ; \delta) \subset \mathcal{V}$.

In view of (3.3), the expansion in the one-dimensional case reads for a smooth function $v$ as

$$
L_{1, \delta} v=\frac{9 K}{5} v^{\prime \prime}+\frac{\ell_{4,1, \delta}}{12} v^{(4)}+\frac{\ell_{6,1, \delta}}{360} v^{(6)}+\cdots
$$

Some straightforward but tedious calculations of the integrals appearing lead for the two-dimensional case e.g. to

$$
L_{2, \delta}^{(4)} \boldsymbol{v}=\frac{\ell_{4,2, \delta} \pi}{192}\left(\begin{array}{c}
5 \partial^{(40)} v_{1}+4 \partial^{(31)} v_{2}+6 \partial^{(22)} v_{1}+4 \partial^{(13)} v_{2}+\partial^{(04)} v_{1} \\
\partial^{(40)} v_{2}+4 \partial^{(31)} v_{1}+6 \partial^{(22)} v_{2}+4 \partial^{(13)} v_{1}+5 \partial^{(04)} v_{2}
\end{array}\right)
$$

and

$$
\begin{gathered}
L_{2, \delta}^{(6)} \boldsymbol{v}=\frac{\ell_{6,2, \delta} \pi}{9216}\left(\begin{array}{c}
7 \partial^{(60)} v_{1}+6 \partial^{(51)} v_{2}+15 \partial^{(42)} v_{1}+12 \partial^{(33)} v_{2}+ \\
\partial^{(60)} v_{2}+6 \partial^{(51)} v_{1}+9 \partial^{(42)} v_{2}+12 \partial^{(33)} v_{1}+ \\
+9 \partial^{(24)} v_{1}+6 \partial^{(15)} v_{2}+\partial^{(06)} v_{1} \\
+15 \partial^{(24)} v_{2}+6 \partial^{(15)} v_{1}+7 \partial^{(06)} v_{2}
\end{array}\right)
\end{gathered}
$$

and for the three-dimensional case e.g. to

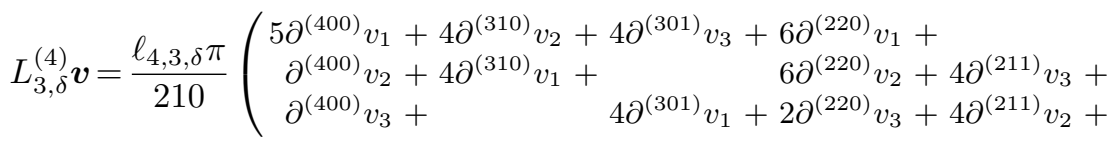

$$
\begin{aligned}
& +6 \partial^{(202)} v_{1}+4 \partial^{(130)} v_{2}+4 \partial^{(121)} v_{3}+4 \partial^{(112)} v_{2}+4 \partial^{(103)} v_{3}+ \\
& +2 \partial^{(202)} v_{2}+4 \partial^{(130)} v_{1}+\quad 4 \partial^{(112)} v_{1}+ \\
& +6 \partial^{(202)} v_{3}+4 \partial^{(121)} v_{1}+\quad 4 \partial^{(103)} v_{1}+
\end{aligned}
$$

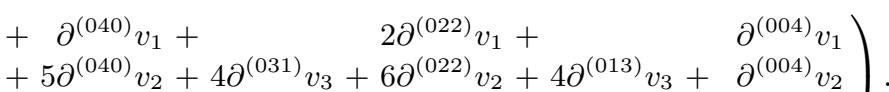

$$
\begin{aligned}
& \left.\begin{array}{l}
+5 \partial^{(040)} v_{2}+4 \partial^{(031)} v_{3}+6 \partial^{(022)} v_{2}+4 \partial^{(013)} v_{3}+\partial^{(004)} v_{2} \\
+\quad \partial^{(040)} v_{3}+4 \partial^{(031)} v_{2}+6 \partial^{(022)} v_{3}+4 \partial^{(013)} v_{2}+5 \partial^{(004)} v_{3}
\end{array}\right) .
\end{aligned}
$$

EXAMPLE 3.3. The linearisation of the proportional microelastic material model (2.4) leads to $\lambda_{d, \delta}(r)=c_{d, \delta} r^{-3}(r<\delta)$ with $c_{d, \delta}$ defined in (2.6). The one-dimensional case cannot be treated here as $\ell_{1, \delta}^{|\cdot|}=\infty$. In the two- and three-dimensional case, the model yields a weakly singular integral kernel of convolution type with $\ell_{d, \delta}^{|\cdot|}=\mathcal{O}\left(\delta^{-2}\right)$. So, all the results of this and the preceding section apply for the two- and three-dimensional case.

The coefficients in the expansion of Theorem 3.2 are calculated from

$$
\ell_{k, d, \delta}=\frac{c_{d, \delta}}{k+d-1} \delta^{k+d-1}, \quad k=2,4, \ldots, \quad d \in\{2,3\} .
$$


Unfortunately, in this model, the interaction jumps to zero if $r=\delta$. This can be avoided by taking $\lambda_{d, \delta}(r)=c_{d, \delta}^{\prime} r^{-3} \exp \left(-\delta^{2} /\left(\delta^{2}-r^{2}\right)\right)(r<\delta)$. The new constant of proportionality $c_{d, \delta}^{\prime}$ is again calculated from (2.10).

It should be noted that Arndt \& Griebel [4, Sect. 4] have obtained, in the onedimensional case, the quite similar expansion (compare with (3.3), (3.6))

$$
\sum_{n=1}^{\infty} \frac{2}{(2 n) !} \varepsilon^{2(n-1)}\left(\phi^{\prime} \circ v\right)^{(2 n)}(x)
$$

for the evolution equation of an atomistic system. Here, $\phi$ is some function describing the potential, e.g. $\phi(r)=(r-1)^{2} / 2$ for a chain of linear springs, and $\varepsilon$ is a scaling parameter. For a further study of crystalline solids, cf. also E \& Ming [15].

The very interesting question whether the sequence of solutions to the peridynamic equation of motion converges in some sense towards a certain solution to the Navier equation cannot be answered so far. In order to find an answer, it might be essential to describe the behavior of the peridynamic solution near the boundary of the domain as one lacks a criterion for posing appropriate boundary conditions for the Navier equation. More precisely, let $\boldsymbol{u}$ be a sufficiently smooth solution to the Navier equation subject to suitable initial and boundary conditions and for a given right-hand side. Then $\boldsymbol{w}_{\delta}:=\boldsymbol{u}_{\delta}-\boldsymbol{u}$ with $\boldsymbol{u}_{\delta} \in \mathcal{C}^{2}\left([0, T] ; L^{2}(\mathcal{V})^{d}\right)$ being a solution to the peridyamic equation with the same initial values and the same right-hand side satisfies for $(\boldsymbol{x}, t) \in \mathcal{V} \times(0, T)$ the equation

$$
\rho(\boldsymbol{x}) \partial_{t}^{2} \boldsymbol{w}_{\delta}(\boldsymbol{x}, t)=\left(L_{d, \delta} \boldsymbol{w}_{\delta}\right)(\boldsymbol{x}, t)+\left(L_{d, \delta} \boldsymbol{u}\right)(\boldsymbol{x}, t)-(L \boldsymbol{u})(\boldsymbol{x}, t)
$$

with initial conditions $\boldsymbol{w}_{\delta}(\cdot, 0)=\partial_{t} \boldsymbol{w}_{\delta}(\cdot, 0)=0$. The uniform stability $(2.18)$ furnishes

$$
\left\|\boldsymbol{w}_{\delta}\right\|_{\mathcal{C}^{1}\left([0, T] ; L^{2}(\mathcal{V})^{d}\right)} \leq C\left\|L_{d, \delta} \boldsymbol{u}-L \boldsymbol{u}\right\|_{L^{2}\left(0, T ; L^{2}(\mathcal{V})^{d}\right)}
$$

with $C$ being independent of $\delta$. With Theorem 3.2, we only have an estimate for $L_{d, \delta} \boldsymbol{u}-L \boldsymbol{u}$ in the interior domain $\mathcal{V}_{0}$ at hand. What would be needed is, however, a characterization of the difference between $L_{d, \delta} \boldsymbol{u}$ and $L \boldsymbol{u}$ near the boundary $\partial \mathcal{V}$.

Acknowledgement. The authors gratefully acknowledge many discussions with Henrik Büsing and Stephan Kusche.

\section{REFERENCES}

[1] B.S. Altan, Uniqueness of initial-boundary value problems in nonlocal elasticity, Int. J. Solids Structures, 25(11), 1271-1278, 1989.

[2] B.S. Altan, Uniqueness in nonlocal thermoelasticity, J. Thermal Stresses, 14, 121-128, 1991.

[3] W. Arendt et al., Vector-Valued Laplace Transforms and Cauchy Problems, Birkhäuser, Basel, 2001.

[4] M. Arndt and M. Griebel, Derivation of higher order gradient continuum models from atomistic models for crystalline solids, Multiscale Model. Simul., 4(2), 531-562, 2005.

[5] Z.P. Bažant and M. Jirásek, Nonlocal integral formulations of plasticity and damage: survey and progress, J. Eng. Mech., 128(11), 1119-1149, 2002.

[6] X. Blanc, C. Le Bris and F. Legoll, Analysis of a prototypical multiscale method coupling atomistic and continuum mechanics, M2AN, 39(4), 797-826, 2005. 
[7] X. Blanc, C. Le Bris and P.L. Lions, From molecular models to continuum mechanics, Arch. Rational Mech. Anal., 164(4), 341-381, 2002.

[8] X. Blanc, C. Le Bris and P.L. Lions, Atomistic to continuum limits for computational materials science, M2AN, 41(2), 391-426, 2007.

[9] F. Bobaru and S.A. Silling, Peridynamic 3D problems of nanofiber networks and carbon nanotube-reinforced composites, Materials and Design: Proc. Numiform, American Institute of Physics, 1565-1570, 2004.

[10] F. Bobaru, S.A. Silling and H. Jiang, Peridynamic fracture and damage modeling of membranes and nanofiber networks, Proc. XI Int. Conf. Fracture, Turin, 5748, 1-6, 2005.

[11] J. Bourgain, H. Brézis and P. Mironescu, Another look at Sobolev spaces, J.L. Menaldi et al. (eds.), Optimal Control and Partial Differential Equations, IOS Press, Amsterdam, Tokyo, 439-455, 2001.

[12] Y. Chen, J.D. Lee and A. Eskandarian, Atomistic viewpoint of the applicability of microcontinuum theories, Int. J. Solids Structures, 41(8), 2085-2097, 2004.

[13] Y. Chen, J.D. Lee and A. Eskandarian, Dynamic meshless method applied to nonlocal crack problems, Theor. Appl. Fracture Mech., 38, 293-300, 2002.

[14] K. Dayal and K. Bhattacharya, Kinetics of phase transformations in the peridynamic formulation of continuum mechanics, J. Mech. Phys. Solids, 54, 1811-1842, 2006.

[15] W. E and P. Ming, Cauchy-Born rule and the stability of crystalline solids: static problems, Arch. Rational Mech. Anal., 183(2), 241-297, 2007.

[16] E. Emmrich and O. Weckner, Analysis and numerical approximation of an integro-differential equation modelling non-local effects in linear elasticity, Math. Mech. Solids, published online first, DOI: 10.1177/1081286505059748, 2005.

[17] E. Emmrich and O. Weckner, The peridynamic equation and its spatial discretisation, Math. Model. Anal., 12 (1), 17-27, 2007.

[18] E. Emmrich and O. Weckner, The peridynamic equation of motion in non-local elasticity theory, C.A. Mota Soares et al. (eds.), III European Conference on Computational Mechanics, Solids, Structures and Coupled Problems in Engineering (Lisbon, June 2006), Springer, 19, 2006.

[19] E. Emmrich, Gewöhnliche und Operator-Differentialgleichungen, Vieweg, Wiesbaden, 2004.

[20] A.C. Eringen, Vistas of nonlocal continuum physics, Int. J. Eng. Sci., 30 (10), 15511565, 1992.

[21] H.O. Fattorini, Second order linear differential equations in Banach spaces, NorthHolland mathematics studies 108, Elsevier, Amsterdam, 1985.

[22] W. Gerstle and N. Sau, Peridynamic modeling of concrete structures, Proc. 5th Int. Conf. Fract. Mech. Concr. Struct, Li et al. (eds.), Ia-FRAMCOS, 2, 949-956, 2004.

[23] W. Gerstle, N. Sau and S. Silling, Peridynamic modeling of plain and reinforced concrete structures, SMiRT 18: 18th Int. Conf. Struct. Mech. React. Technol., Beijing, China, 15, 2005.

[24] E. Kröner, Elasticity theory of materials with long range forces, Int. J. Solids Structures, 3, 731-742, 1967.

[25] I.A. Kunin, Elastic Media with Microstructure, vol. I and II. Springer, Berlin, 1982/83.

[26] C. Le Bris and P.L. Lions, From atoms to crystals: a mathematical journey, Bulletin of the American Mathematical Society, 42 (3), 291-363, 2005.

[27] Y. Lei, M.I. Friswell and S. Adhikari, A Galerkin method for distributed systems with non-local damping, Int. J. Solids Structures, 43(11-12), 3381-3400, 2005.

[28] O.A. Oleinik, A.S. Shamaev and G.A. Yosifian, Mathematical Problems in Elasticity and Homogenization, North-Holland Publishing, Amsterdam, 1992.

[29] A.A. Pisano and P. Fuschi, Closed form solution for a nonlocal elastic bar in tension, Int. J. Solids Structures, 40 (1), 13-23, 2003.

[30] C. Polizzotto, Nonlocal elasticity and related variational principles, Int. J. Solids Struc- 
tures, 38 (42-43), 7359-7380, 2001.

[31] C. Polizzotto, Unified thermodynamic framework for nonlocal/gradient continuum mechanics, Eur. J. Mech. A/Solids, 22, 651-668, 2003.

[32] D. Rogula, Nonlocal Theory of Material Media, Springer, Berlin, 1982.

[33] S.A. Silling, Reformulation of elasticity theory for discontinuities and long-range forces, J. Mech. Phys. Solids, 48 (1), 175-209, 2000.

[34] S.A. Silling, Dynamic fracture modeling with a meshfree peridynamic code, K.J. Bathe (ed.), in Computational Fluid and Solid Mechanics 2003, Elsevier, Amsterdam, 641-644, 2003.

[35] S.A. Silling and E. Askari, Peridynamic modeling of impact damage, F.J. Moody (ed.), Amer. Soc. of Mech. Eng., New York, 489, 197-205, 2004.

[36] S.A. Silling and E. Askari, A meshfree method based on the peridynamic model of solid mechanics, Comput. Struct., 83(17-18), 1526-1535, 2005.

[37] S.A. Silling and F. Bobaru, Peridynamic modeling of membranes and fibers, Int. J. Nonlinear Mech., 40, 395-409, 2005.

[38] S.A. Silling, M. Zimmermann and R. Abeyaratne, Deformation of a peridynamic bar, J. Elasticity, 73, 173-190, 2003.

[39] R. Temam and A. Miranville, Mathematical Modeling in Continuum Mechanics, Cambridge University Press, 2001.

[40] J. Wang and R.S. Dhaliwal, Uniqueness in generalized nonlocal thermoelasticity, J. Thermal Stresses, 16, 71-77, 1993.

[41] J. Wang and R.S. Dhaliwal, On some theorems in the nonlocal theory of micropolar elasticity, Int. J. Solids Structures, 30(10), 1331-1338, 1993.

[42] O. Weckner and R. Abeyaratne, The effect of long-range forces on the dynamics of a bar, J. Mech. Phys. Solids, 53(3), 705-728, 2005.

[43] O. Weckner and E. Emmrich, Numerical simulation of the dynamics of a nonlocal, inhomogeneous, infinite bar, J. Comp. Appl. Mech., 6(2), 311-319, 2005.

[44] J. Wloka, Partial Differential Equations, Cambridge University Press, 1987.

[45] M. Zimmermann, A Continuum Theory With Long-Range Forces for Solids, PhD Thesis, Massachusetts Institute of Technology, Department of Mech. Engineering, 2005. 